\title{
Availability of palliative care services in Zambia: A nationwide provincial and tertiary hospital survey
}

\author{
Brigitte Frett Utter ${ }^{\mathrm{a}}$, Groesbeck P. Parham ${ }^{\mathrm{b}, \mathrm{d}}$, Claire-Helene Mershon ${ }^{\mathrm{a}}$, Kombatende Sikombe ${ }^{\mathrm{a}}$, \\ Agnes Musonda $^{\mathrm{a}}$, Jane Matambo ${ }^{\mathrm{a}}$, Sharon Kapambwe ${ }^{\mathrm{a}}$, S. Citonje Msadabwe ${ }^{\mathrm{c}}$, Katundu Katundu ${ }^{\mathrm{a}}$, \\ Allen Bateman ${ }^{\mathrm{a}, \mathrm{b}}$, Kennedy Lishimpi ${ }^{\mathrm{c}}$, Mulindi Mwanahamuntu ${ }^{\mathrm{d}}$, Carla J. Chibwesha ${ }^{\mathrm{a}, \mathrm{b}, *}$ \\ ${ }^{a}$ Centre for Infectious Disease Research in Zambia (CIDRZ), P.O. Box 34681, Lusaka, Zambia \\ ${ }^{\mathrm{b}}$ Division of Global Women's Health, Department of Obstetrics and Gynecology, University of North Carolina at Chapel Hill School of Medicine, 3009 Old Clinic Building \\ CB \#7570, Chapel Hill, NC, 27599-7570, USA \\ ${ }^{\mathrm{c}}$ Cancer Diseases Hospital, Lusaka, Zambia \\ ${ }^{\mathrm{d}}$ University of Zambia, P.O. Box 32379, Lusaka, Zambia
}

\section{A R T I C L E I N F O}

Keywords:

Palliative care

Sub-Saharan Africa

Zambia

\begin{abstract}
A B S T R A C T
Background: With high rates of HIV/AIDS and rising burdens of non-communicable diseases, Zambians increasingly need palliative care. While efforts are underway to grow Zambia's palliative care system, the most recent situational analysis of palliative care in Zambia, conducted in 2008, revealed substantial gaps in availability.

Methods: To provide an updated appraisal of breast and cervical cancer services in Zambia, including palliative care, we conducted a nationwide provincial and tertiary hospital survey. All 9 provincial hospitals and the University Teaching Hospital and Cancer Diseases Hospital in Lusaka Province participated $(\mathrm{N}=11)$. The survey was conducted between August 2014 and January 2015 and administered in-person at each facility. Data regarding the availability of inpatient, outpatient, and community-based palliative care services, palliative medications, and psychosocial supports was obtained at each facility. The reported results are descriptive in nature. Results: Although the need for palliative care services was recognized, many facilities (64\%) lack palliative care policies and only $18 \%$ offer palliative care in a coordinated program. The majority of services are only available to inpatients and rarely include community-based programs. While all facilities had adequate supplies of acetaminophen, $82 \%$ reported unavailability of codeine and $45 \%$ reported no access to oral morphine.

Conclusions: This assessment confirms the dearth of palliative care services across Zambia. Less than half of its provincial hospitals offer community- or home-based services and only 55\% offer opioid analgesics. Immediate and substantial improvements in policy, drug procurement and distribution, and service expansion are needed to ensure high-quality palliative care is available throughout Zambia.
\end{abstract}

\section{Introduction}

Palliative care emphasizes improving quality of life through medical, psychological, and spiritual services. These approaches are applicable to many diseases including cancer and HIV/AIDS [1,2]. Globally, palliative care models are associated with positive outcomes including reduced symptom burden, increased quality of life, and improved psychosocial wellbeing [3-6]. Unfortunately, the global need for services far exceeds availability, this is especially true throughout sub-Saharan Africa. While efforts to increase palliative care services across Africa have led to improvements in care in the last 12 years, the majority of African countries (52\%) lack organized palliative care

Abbreviations: CDH, cancer diseases hospital; HFA, health facility assessment; PCAZ, Palliative Care Association of Zambia; WHO, World Health Organization

* Corresponding author at: Division of Global Women's Health, Department of Obstetrics and Gynecology, University of North Carolina at Chapel Hill, 2009 Old

Clinic Building, Chapel Hill, BC 27599.

E-mail addresses: frettb@gmail.com (B. Frett Utter), gparham@med.unc.edu (G.P. Parham), clairehelene.mershon@gmail.com (C.-H. Mershon), kombatende.sikombe@cidrz.org (K. Sikombe), agnes.musonda@cidrz.org (A. Musonda), jane.matambo@cidrz.org (J. Matambo), sharon.kapambwe@cidrz.org (S. Kapambwe), citonje@yahoo.com (S.C. Msadabwe), katundu.katundu@gmail.com (K. Katundu), bateman.allen@gmail.com (A. Bateman), kcmlishimpi@yahoo.co.uk (K. Lishimpi), mulindim@hotmail.com (M. Mwanahamuntu), carla_chibwesha@med.unc.edu (C.J. Chibwesha). 
services [7].

In Zambia, there is a generalized HIV/AIDS epidemic, the cervical cancer rate is the $5^{\text {th }}$ highest in the world, and breast cancer is routinely diagnosed at advanced stages. Such a setting demands comprehensive palliative care services $[8,9]$. Although momentum is building, research indicates Zambia's palliative care infrastructure remains weak $[10,11]$.

According to the World Health Organization's Public Health Strategy for expanding palliative care, key elements of national palliative care programs include: policies, drug availability, education, and service implementation [12]. To obtain an overview of the availability of palliative care in Zambia, we completed an analysis of results from a nationwide, structured assessment documenting the availability of breast and cervical cancer screening, treatment, and care services in Zambia, including palliative care. The primary findings from this assessment as well as an overview of the methodology utilized were published in this journal in 2017 [8]. Adding to that publication, we report here our findings s pecific to palliative ca re, us ing the WHO Public Health Strategy as a benchmark.

\section{Methods}

This analysis utilizes data from a facility assessment survey completed at all 9 provincial hospitals as well as the University Teaching Hospital and Cancer Diseases Hospital in Lusaka $(\mathrm{N}=11)$. The survey methods are described in detail in our prior publication of the primary findings [8]. Briefly, facilities were chosen due to their priority listing for service expansion in the Zambian National Cancer Control Strategic Plan. At each facility, we administered an in-person survey to collect self-reported data on human resources and facility infrastructure for providing breast and cervical cancer services, including palliative care. We also conducted a structured interview with each Provincial Health Officer. A member of our research team traveled to each facility to administer the survey and conduct key informant interviews.

The survey, developed by our team, examined three domains. The first was the availability of and demand for palliative care. The availability domain included surveying facilities on the number and types of healthcare providers available at that location as well as the availability of specific services. As part of this domain, we also evaluated whether patients seek palliative care and the settings where services are available. The second domain was an assessment of availability of psychosocial and spiritual support for patients and families. The third domain was the accessibility of palliative care medications. Medications were selected from the International Association for Hospice and Palliative Care List of Essential Medicines for Palliative Care. Stock-outs of key medications were evaluated via self-report by asking: (1) in-stock today, (2) out of stock today, (3) out of stock for 7 days, (4) out of stock for 30 days, (5) out of stock for 90 days, (6) not available, or (7) don't know.

Descriptive analyses were performed using Microsoft Excel 2010 (Redmond, WA, U.S.A.). Regulatory approval was provided by the Biomedical Research Ethics Committee of the University of Zambia (Ref no. 009-06-14) and the Zambian Ministry of Health. Approvals included a waiver of informed consent as our unit of analysis was the facility.

\section{Results}

Between August 2014 and January 2015, 11 Zambian hospitals participated in our study. Our survey revealed a national average of 2 healthcare workers per 10,000 population, an estimate devised from the total number of clinical providers (physicians, nurses, midwives, pharmacists, and clinical officers) and population estimates. Eight facilities $(72 \%)$ reported a demand for palliative care, with $4(36 \%)$ reporting that patients often present seeking palliative care, $1(9 \%)$ reporting that patients sometimes present seeking palliative care, and 3 (27\%) reporting that patients rarely present seeking palliative care. While $8(72 \%)$ facilities provide inpatient palliative care, only $2(18 \%)$ provide coordinated services. Outpatient and home-based services are offered by $4(36 \%)$ and $3(27 \%)$ of the facilities, respectively. Only $36 \%$ of facilities reported having a policy for palliative care delivery.

As part of the service availability domain, we also assessed for availability of counseling. All 11 facilities reported that they offer psychosocial counseling, with $9(82 \%)$ reporting staff trained in counseling or bereavement but only $1(9 \%)$ reporting that counseling is provided by a psychiatrist or psychologist. The most common providers of psychological counseling were nurses or midwives. Additionally, half (55\%) of facilities surveyed had chaplains. The final aspect of the service domain assessed was whether facilities offer housing for patients receiving palliative care services and the availability of communitybased care. While $10(91 \%)$ facilities offer housing for palliative care patients, only 2 (18\%) offer community-based programs.

Under the medication domain, we assessed whether medications indicated for (1) pain, (2) nausea/vomiting, (3) anorexia, and (4) depression, anxiety, delirium, psychosis, and/or seizure were available. All facilities reported that acetaminophen was available the day of the survey, while $2(18 \%)$ indicated that ibuprofen was out of stock. Seven (64\%) facilities reported that codeine was never available, while $45 \%$ reported that morphine had been out of stock for 90 days. Most facilities $(10 ; 91 \%)$ reported that fentanyl, methadone, and oxycodone were never available while seven $(64 \%)$ indicated that senna was never available. Three (27\%) facilities reported stock-outs of dexamethasone and prednisone, 1 (9\%) reported stock-outs of carbamazepine, and 6 (55\%) reported stock-outs of amitriptyline.

\section{Discussion}

The results of our health facility assessment reveal major gaps and opportunities for improving the availability of palliative care services across Zambia. As noted, only $36 \%$ of facilities reported having a policy governing the provision of palliative care, highlighting the need for national guidance on palliative care development throughout the country. While inpatient palliative care services are accessible at the majority of provincial-level facilities in Zambia, few offer outpatient or home-based services. This is concerning for a number of reasons. First, long distances and travel times to health facilities are known to affect health-seeking behaviors [13,14]. Second, integrating palliative care into communities is essential for expanding service availability. For example, trained family members can meet the majority of palliative care needs for patients at home and communities can take ownership of palliative care programming, promoting sustainability [1]. There are several models that can be considered as Zambia expands access to community-based services, including community health worker models currently in use in Malawi [2].

Our findings also indicate that inpatient services should be expanded to meet a growing burden of chronic disease. While inpatient palliative care is increasingly available in Zambia, it is not yet available at all provincial-level facilities [10]. Given that $80 \%$ of cancer diagnoses occur at advanced stages in developing countries and the cancer burden continues to rise, the need for inpatient services will expand and building capacity must be prioritized. Access to palliative care medications was also limited, including for inpatients. The WHO "pain ladder" has been shown to provide adequate pain relief for most patients [15]. According to the ladder, the first step is to attempt pain control with non-opioid medications. If pain remains untreated, oral codeine can be used, followed by oral morphine. Unfortunately, the majority of facilities surveyed reported that codeine is either never available or was out-of-stock on the day of the survey. Additionally, oral morphine was unavailable at nearly half of facilities. Since these medications can be cheaply manufactured and procured, availability must be addressed through centrally organized procedures.

To our knowledge, our survey is the first published assessment of the availability of palliative care in Zambia that includes information from each of Zambia's provinces. It therefore provides a nationwide 
appraisal of service availability and clarifies priority areas for future programming. Although our survey covered the critical components of WHO's Public Health Strategy as it relates to palliative care, it was limited in that only provincial and tertiary hospitals were included. As such, our findings $\mathrm{m}$ ay $\mathrm{n}$ ot be g eneralizable to lower-level facilities. Importantly, all data were self-reported and none of the reported information was verified through observation of patient-provider interactions or pharmacy stocks. This aspect of the study design may have introduced recall and reporting bias.

\section{Conclusion}

Palliative care is an essential healthcare service. Our findings demonstrate that while inpatient palliative care services are accessible at $72 \%$ of provincial-level facilities in Zambia, few facilities offer outpatient or home-based services. Access to palliative care medications is also limited. Implementation of a national framework that outlines guidance for (1) inpatient, outpatient, and home-based services, (2) educational programs, and (3) procurement of palliative care medications will be an important first step in expanding palliative care. Such a national policy will lay the groundwork for program development, ensuring that access to palliative care can expand throughout Zambia.

\section{Authors' contributions}

BF and CJC conceived of and designed the study, performed the analyses, and drafted the manuscript. GPP conceived of the study and provided critical input on the manuscript. CHM, KS, AM, JM, SK, and $\mathrm{AB}$ participated in the study design, the acquisition of data, and provided critical input on the manuscript. SCM, KK, MM, and KL reviewed and provided critical input on the manuscript. All authors approved the final version of the manuscript for publication.

\section{Declarations of interest}

None.

\section{Acknowledgements}

Special thanks to all survey respondents from the participating hospitals and provincial health offices who gave generously of their time in responding to our questionnaire.

The nationwide facility assessment survey was funded by the Susan G. Komen Foundation Award GSP14UNC006. Additional investigator support was provided through a Fulbright-Fogarty Fellowship Award, the National Cancer Institute Award 1D43CA153784, and the Fogarty
International Center Award R25TW009340 to the UNC Hopkins Morehouse Tulane Fogarty Global Health Fellows Program. The funders had no role in the study design, data collection, data analysis and interpretation, manuscript writing, or in the decision to submit the manuscript for publication.

\section{References}

[1] Worldwide Palliative Care Alliance and WHO, Global Atlas of Palliative Care at the End of Life, (2014) Accessed 28 February 2018 http://www.thewhpca.org/ resources/global-atlas-on-end-of-life-care.

[2] M.E. Herce, S.N. Elmore, N. Kalanga, J.W. Keck, E.B. Wroe, A. Phiri, et al., Assessing and responding to palliative care needs in rural Sub-Saharan Africa: results from a model intervention and situational analysis in Malawi, PLoS One 9 (10) (2015) e110457.

[3] D.E. Meier, O.W. Brawley, Palliative care and the quality of life, J. Clin. Oncol. 29 (20) (2011) 2750-2752.

[4] G. Vanbutsele, K. Pardon, S. Van Belle, V. Surmont, M. De Laat, R. Colman, et al., Effect of early and systematic integration of palliative care in patients with advanced cancer: a randomized controlled trial, Lancet Oncol. 19 (3) (2018) 394-404.

[5] K. Lowther, L. Selman, V. Simms, N. Gikaara, A. Ahmed, A. Zipporah, et al., Nurseled palliative care for HIV-positive patients taking antiretroviral therapy in Kenya : a randomised controlled trial, Lancet HIV 2 (8) (2015) E328-E334.

[6] J. Bates, T. Mphwatiwa, J. Ardrey, N. Desmond, L.W. Niessen, S.B. Squire, Household concepts of wellbeing and the contribution of palliative care in the context of advanced cacner: a photovoice study from Blantyre, Malawi, PLoS One 13 (8) (2018) e0202490.

[7] J.Y. Rhee, E. Garralda, C. Torrado, S. Blanco, I. Ayala, E. Namisango, et al., Palliative care in Africa: a scoping review from 2005-16, Lancet Oncol. 18 (9) (2017) e522-e531.

[8] C. Chibwesha, L.F. Pinder, A. Musonda, K. Sikombe, J. Matambo, A.C. Bateman, et al., A comprehensive assessment of breast and cervical cancer control infrastructure in Zambia, J. Cancer Policy 13 (2017) 24-29.

[9] M.H. Mwanahamuntu, V.V. Sahasrabuddhe, K.S. Pfaendler, V. Muenda, M.L. Hicks, S.H. Vermund, et al., Implementation of 'see-and-treat' cervical cancer prevention services inked to HIV care in Zambia, AIDS 23 (March (6)) (2009) N1-N5.

[10] F.M. Goma, W. Mutale, S. Mkwananzi, Countrywide Situational Report on Palliative Care in Zambia by the Palliative Care Association of Zambia, (2008) Accessed 14 April 2015 http://www.pcaz.org.Zm/PDF\%20Folder/PCAZ-FINAL $\% 20$ SITUATIONAL\%20ANALYSIS.pdf.

[11] J.Y. Rhee, E. Garralda, E. Namisango, E. Luyirika, L. de Lima, R.L. Powell, et al., An analysis of palliative care development in Africa: a ranking based on region-specific macro indicators, J. Pain Symptom Manage. 56 (2) (2018) 230-238, https://doi. org/10.1016/j.jpainsymman.2018.05.005.

[12] J. Stjernsward, K.M. Foley, F.D. Ferris, The Public Health Strategy for palliative care, J. Pain Symptom Manage. 33 (5) (2007) 486-493.

[13] B.I. Eze, J.N. Eze, Demographics, socio-economic and clinical characteristics: implications for time to presentation at a Nigerian tertiary ophthalmic outpatient population, Public Health 128 (2014) 1023-1029.

[14] S. Gabrysch, S. Cousens, J. Cox, O.M. Campbell, The influence of distance and level of care on delivery place in rural Zambia: a study of linked national data in geographic information system, PLoS Med. 8 (1) (2011) e1000394.

[15] WHO, National Cancer Control Programmes: Policies and Managerial Guidelines, 2nd edition, (2002) Accessed 17 July 2017 http://www.who.int/cancer/ publications/nccp2002/en/. 